\title{
INFECTION ASSOCIATED HEMOPHAGOCYTIC SYNDROME IN CHILDREN: A RETROSPECTIVE ANALYSIS OF 17 CASES
}

\section{$\underline{\text { Meltem Polat }}^{1}$, Fatma Burcu Belen ${ }^{2}$}

${ }^{1}$ Pamukkale University School of Medicine, Department of Pediatric Infectious Diseases, Denizli, Turkey

${ }^{2}$ Gaziantep Childrens' Hospital, Department of Pediatric Hematology, Gaziantep, Turkey

\section{Background}

Haemophagocytic syndrome (HPS) is a rapidly progressive, life-threatening syndrome of excessive immune activation. Early recognition of the clinical presentations and laboratory abnormalities associated with HPS and prompt initiation of treatment can be life saving. The aim of this study was to investigate the infectious etiologies, clinical and laboratory characteristics, and treatment outcomes of pediatric patients with infection associated hemophagocytic syndrome (IAHPS).

\section{Methods}

This is a retrospective record review of pediatric patients diagnosed to have IAHFS between December 2012 and January 2016.
A diagnosis of HFS was assigned by means of the HLH guidelines (HLH-94).

\section{Results}

A total of 17 pediatric patients with IAHPS (10 boys / 7 girls; median age: 6 years, range 2-17 years) were enrolled in this study. Brucella (10 patients, 58\%) was the most frequent infectious trigger of HFS. Other infectious triggers were Leishmania (3 patients), Salmonella Typhi (1 patient), Mycobacterium tuberculosis (1 patient), parvovirus B19 (1 patient), and influenza A (H3N2) (1 patient). Fever, bi/pansitopenia, hyperferritinemia, and elevated transaminases were present in all patients (100\%), and splenomegaly in 13 patients $(76 \%)$. The median duration of fever before diagnosis of HPS was 12 days (range, 5-25 days). All of the patients underwent bone marrow aspirations that confirmed hemophagocytosis. All patients received antimicrobial treatment for the underlying infections; 10 patients received intravenous immunoglobulin (IVIG) and 2 patients received IVIG+steroids due to persistent fever and progressively worsening clinical conditions. Recovery was noted in 16 (94\%) patients. Mortality was only seen in 1 patient (5.8\%) with influenza A (H3N2) pneumonia.

\section{Conclusions}

Physicians need to be aware of the occurrence of HFS in patients with prolonged fever, organomegaly, and cytopenias in the setting of an infectious process. 